\title{
Immigrant Latino parents demonstrated high interactivity with pediatric primary care text messaging intervention
}

\author{
Luke G. Silverman-Lloyd ${ }^{1}$, Jose Dominguez Cortez ${ }^{2}$, Sashini K. Godage ${ }^{3}$, Doris Valenzuela Araujo ${ }^{4}$, \\ Tatiahna Rivera ${ }^{5}$, Sarah Polk ${ }^{2,6}$, Lisa Ross DeCamp ${ }^{7}$ \\ ${ }^{1}$ University of California, Berkeley-University of California, San Francisco Joint Medical Program, Berkeley, CA, USA; ${ }^{2}$ Johns Hopkins Center \\ for Health/Salud and Opportunity for Latinos, Baltimore, MD, USA; ${ }^{3}$ Harvard Medical School, Boston, MA, USA; ${ }^{4}$ Oregon Health \& Science \\ University School of Medicine, Portland, OR, USA; ${ }^{5}$ Loyola University Chicago, Stritch School of Medicine, Maywood, IL, USA; ${ }^{6}$ Department \\ of Pediatrics, Johns Hopkins University School of Medicine, Baltimore, MD, USA; ${ }^{7}$ Department of Pediatrics, University of Colorado School of \\ Medicine and Children's Hospital Colorado, Aurora, CO, USA
}

Contributions: (I) Conception and design: LR DeCamp, S Polk; (II) Administrative support: SK Godage, D Valenzuela Araujo, T Rivera; (III) Provision of study materials or patients: SK Godage, D Valenzuela Araujo, T Rivera; (IV) Collection and assembly of data: LG Silverman-Lloyd, S. Godage, D Valenzuela Araujo, T Rivera, J Dominguez Cortez; (V) Data analysis and interpretation: LG Silverman-Lloyd, J Dominguez Cortez, LR DeCamp; (VI) Manuscript writing: All authors; (VII) Final approval of manuscript: All authors.

Correspondence to: Lisa Ross DeCamp, MD, MSPH. ACCORDS (Adult and Child Consortium for Health Outcomes Research and Delivery Science), University of Colorado I Children's Hospital Colorado, 13199 E Montview Blvd, Suite 300, Mail Stop F443, Aurora, CO 80045, USA. Email: lisa.decamp@childrenscolorado.org.

Background: The majority of Latino immigrants have been shown to have a mobile phone. Cellular phones offer a low-cost method of reaching larger populations and have the potential for increased tailoring and interactivity. This supports the development of mHealth interventions to address healthcare disparities in this population. In this study we sought to evaluate the feasibility and acceptability of interactive Spanishlanguage text messages sent throughout a child's first year of life in a low-income, limited-English proficient (LEP) Latino population to support families in accessing and using pediatric primary care more effectively.

Methods: Participants $(n=79)$ received interactive text messages over a period of 12 months as a part of a multi-modal mHealth intervention conducted at an urban academic pediatric primary care practice. Inclusion criteria were: singleton infant $<2$ months of age, enrollment in public health insurance, parent age $>18$, parent preferred healthcare language of Spanish, and at least one household cellular phone. Interactive text messages were designed to promote increased healthcare engagement and prompted participant responses through preprogramed algorithms. Text message sequences included clinic appointment reminders, support for obtaining medicine and completing referral appointments, monitoring of illness care needs and use, and parent support program reminders. Descriptive analyses were used to examine text message volume, usability, and participant response to text sequences.

Results: Among participants, mean parent age was 30.1 years (SD: 6.1 years); mean years in the US was 7.5 years (SD: 5.1 years). $63.3 \%$ of parents had less than a high school education and $84.8 \%$ of parents had possible/high likelihood of limited health literacy. Participants completed the majority of sequences with appointment reminder sequences having the quickest response time. The top quartile of responders completed $88.3 \%$ of sequences; lower educational attainment was associated with lower text message sequence completion. Participants rated the program positively, especially the appointment reminders.

Conclusions: LEP Latino parents successfully engaged with interactive Spanish-language text sequences and parent acceptability was high. This study demonstrates feasibility for interventions employing this technology. Text message interventions may be a feasible approach to reduce healthcare disparities and costs for vulnerable populations.

Keywords: Text messaging; mHealth; Latino; healthcare disparities; immigrant 
Received: 21 September 2019; Accepted: 16 January 2020; Published: 05 October 2020.

doi: 10.21037/mhealth.2020.01.06

View this article at: http://dx.doi.org/10.21037/mhealth.2020.01.06

\section{Introduction}

Latinos comprise the largest share of the 44 million immigrants in the US, and Spanish is the most common language spoken among the 25 million people in the US with limited English proficiency (LEP). Latino children in immigrant families experience disparities in healthcare access and quality (1-3). Language and cultural barriers, unfamiliarity with the US healthcare system, and low parental health literacy are barriers to effective healthcare access and use for Latino children (4-8). Health systems also face challenges in providing equitable care for immigrant patients and families (9-11).

Immigrant and LEP patient populations have derived fewer benefits from mHealth interventions, compared with non-immigrant populations $(12,13)$. In the last decade, health systems have focused significant resources on developing and implementing patient portals both on the web and through smartphone applications to increase access to healthcare services (14). Use of patient portals, however, has been comparatively lower among racial/ethnic minority and low-income populations (15-17). Alternatives to patient portals should be considered to promote digital engagement across diverse patient populations.

mHealth interventions utilizing text messaging have shown efficacy in improving appointment adherence, increasing vaccination, and decreasing ED use among primary care populations (18-22). mHealth interventions often exclude non-English speakers and immigrant populations, although some have included Spanish language materials or messages. Text4baby, a perinatal health education program delivered through passive educational text messages, demonstrated success at reaching lowincome Spanish-speaking parents with positive user assessments (23). Another study using text messaging as an adjunct to cognitive behavioral therapy (CBT) in a lowincome population showed positive engagement and user assessments among both English- and Spanish-speakers (24).

In text message interventions (TMIs), text messages can be tailored to individual patients and allow for interaction between the patient and clinic. Text message tailoring and interactivity have been found to increase the efficacy of health engagement and promotion interventions
$(18,25)$. There is limited information on the feasibility and implementation of these strategies among immigrant and LEP patient populations, however $(26,27)$. In this study, we sought to evaluate the feasibility and acceptability of interactive text messages sent throughout a child's first year of life in a low-income, LEP Latino population to support families in accessing and more effectively using pediatric primary care.

\section{Methods}

\section{Study population and setting}

The short message service (SMS) messages delivered in this study (hereafter referred to as text messages) were part of a randomized controlled trial evaluating a multi-modal intervention to support healthcare navigation by LEP Latino parents of infants. Intervention participants received video-based education at the onset of the study, followed by the delivery of a series of interactive text messages throughout the child's first year of life to prompt and assess the parent's healthcare engagement and health-promoting behaviors.

Participants were recruited between February and October 2016 at an urban, academic general pediatrics clinic in the US with approximately 11,000 visits per year. The clinic's majority patient population is publicly insured, Latino children with LEP immigrant parents. Clinic services are tailored to meet the particular sociocultural needs of Latino immigrant families. Clinic providers include board-certified pediatricians, resident physicians, and a pediatric nurse practitioner. Nearly all providers at the clinic communicate directly with patients and parents in Spanish, for whom this is their preferred healthcare language. All providers who communicate directly in Spanish have had their language proficiency assessed according to health system policy.

Participants in this study were parents or legal guardians of publicly insured, singleton US-born infants under 2 months of age. Additional inclusion criteria were: minimum respondent age of 18 years, self-identification of Latino/a, foreign-born, preferred healthcare language of Spanish, and at least one working cellular phone in 
the household. Study participants were recruited by one of two methods: in-person during a scheduled well visit at the clinic or via a letter and follow-up phone call to parents of potentially eligible infants. Parents completed an enrollment survey before the infant was 55 days old after an informed consent process. The consent form was orally read to them and understanding was ascertained. After completion of the enrollment survey, allocation to the intervention or usual care arm of the RCT was unmasked to participants. Randomization was performed by computer random number generation in blocks of ten to balance allocation over time. Participants in the intervention arm were subsequently oriented to the text-messaging portion of the study and began to receive intervention text messages. At their child's 2-month well child visit, participants randomized to the intervention group completed a videobased education and a study team member answered any remaining questions about the text message component.

The Institutional Review Board at Johns Hopkins Medicine approved this study. Intervention participants received $\$ 90$ for their participation. This included $\$ 30$ for the enrollment and final survey (age 12-15 months), $\$ 10$ for the midpoint phone survey (age 7-9 months) and \$20 to provide support for any related cellular phone charges as participants' personal phones were used for delivery of text messages. This study was registered at clinicaltrials.gov (NCT02647814).

\section{Text message development process and content}

Interactive text messages that prompted and assessed healthcare engagement-promoting behaviors were programmed and delivered via emocha ${ }^{\circledR}$, an open-source, secure, HIPAA compliant, and modifiable mHealth application. emocha ${ }^{\circledR}$ allowed for the use of tailored "smart forms" to generate interactivity through delivery of messages and automated responses via pre-programmed algorithms (28). Text message content was developed based on a situated Information, Motivation, Behavioral theorybased (sIMB) framework, which has been used previously to understand and intervene on patient engagement (29). Text messages were developed in conjunction with the Spanishlanguage patient/family advisory council for the study clinic to ensure messages conveyed a positive, motivating tone, wording was clear, and response actions were understood. Bright Futures and the corresponding Family Voices guide were used to ensure consistency with anticipatory guidance and education recommended by the American Academy of
Pediatrics and to develop family-centered wording $(30,31)$.

Text messages sent to participants were characterized as either sequences or push messages. Sequences were formatted as questions intended to prompt replies; they were automated, interactive, and varied in length (Table 1). Four different sequence types were sent to participants during the study: appointment reminders (APT), support for obtaining prescription medicines (MED), support for completing specialty referrals (REF), and monitoring of illness care needs and use and education about use of primary care instead of the emergency department for routine childhood illness (ICM). APT, MED and REF sequences were coordinated around well child appointments, occurring at 2, 4, 6, 9 and 12 months after the child's date of birth (DOB). APT sequences were sent two days prior to each scheduled appointment. MED sequences were sent 2 days after, and REF sequences 5 days after each scheduled appointment. ICM sequences were sent at 100, 220 and 340 days after a child's DOB. Push messages were used for parent education and support and did not prompt replies. Three different types of push messages were sent during the study: flu vaccine reminders, parent support program reminders (SUP) and public benefit program reminders (PUB) consisting of information for applying for food stamps, and insurance renewal reminders. Figure 1 displays a timeline of text messages delivered during the intervention.

\section{Measures}

Parent RCT enrollment surveys were conducted by bilingual research assistants at the recruitment visit. Mid-point and follow-up surveys, during which intervention participants reported on their text messaging experiences, were administered between 7-9 months and 12-15 months of age, respectively. Survey responses were captured simultaneously with survey administration via recording of responses onto a touchscreen tablet computer using REDCap (Research Electronic Data Capture) software $(32,33)$.

Parent information obtained during RCT enrollment surveys included: parent age, race/ethnicity, educational attainment, country of origin, length of stay in the US, family income, English language proficiency, health literacy, family structure and birth history. English proficiency was assessed using the US Census Bureau question, "How well do you speak English?” (34). Parent health literacy was measured using the Spanish-language version of the Newest Vital Sign (NVS), a valid and reliable tool 
Table 1 Example text messages from a multi-modal RCT evaluating an intervention to support healthcare navigation by LEP Latino parents of infants

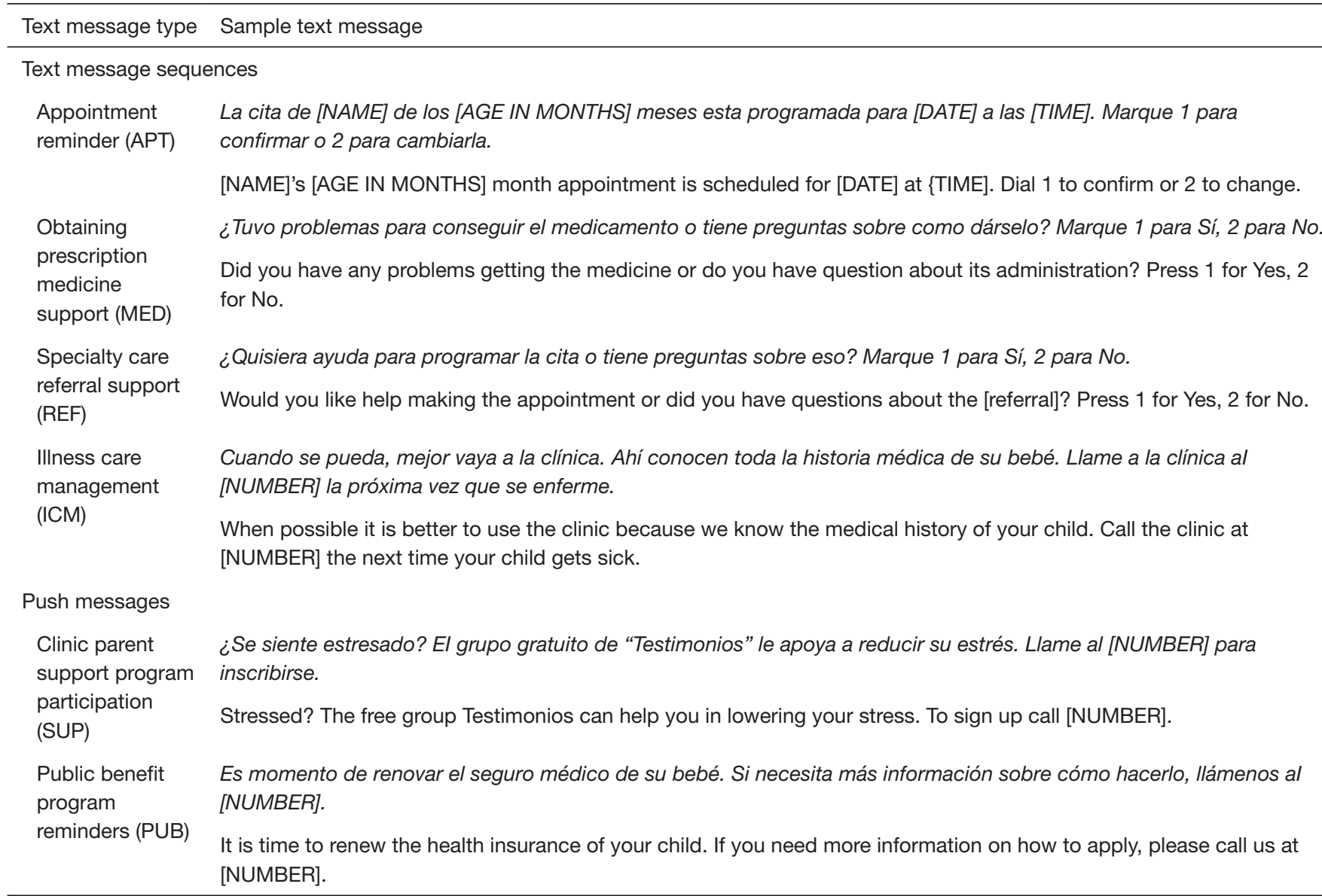

developed by Pfizer Corporation to screen for low health literacy in clinical settings (35). Scores on the NVS correspond to three categories of health literacy based on the number of correct answers: Limited [0-1], Marginal [2-3], and Adequate [4-6]. Parents were also asked to report smartphone use, home internet access, method of internet access, cellular phone plan type and their child's health status.

For the text message intervention, we measured the total number of messages sent and received by all participants, as well as frequency of error message generation. Error messages resulted when participants responded with any message that did not correspond to one of our system's preprogrammed accepted response values. For text message sequences, we measured frequency of responses, response time, and frequency of sequence completion. To assess for subgroup differences in interactivity with the text message intervention, we categorized frequency of sequence completion to create a variable to measure participant engagement. Participants in the upper quartile of frequency of sequence completion were categorized as having "high engagement", while those in the lowest quartile of sequence completion were categorized as having "low engagement".

\section{Analysis}

Parent and child characteristics were assessed using means and percentages. Frequency of message response and sequence completion were assessed using percentages. Response time to initial sequence messages was assessed in minutes using medians and interquartile ranges. For response times, we focused solely on the time it took participants to respond to the initial message of the sequence and not on subsequent sequence messages, as response times to subsequent messages lacked variability. We used chi-square statistics to assess for differences 


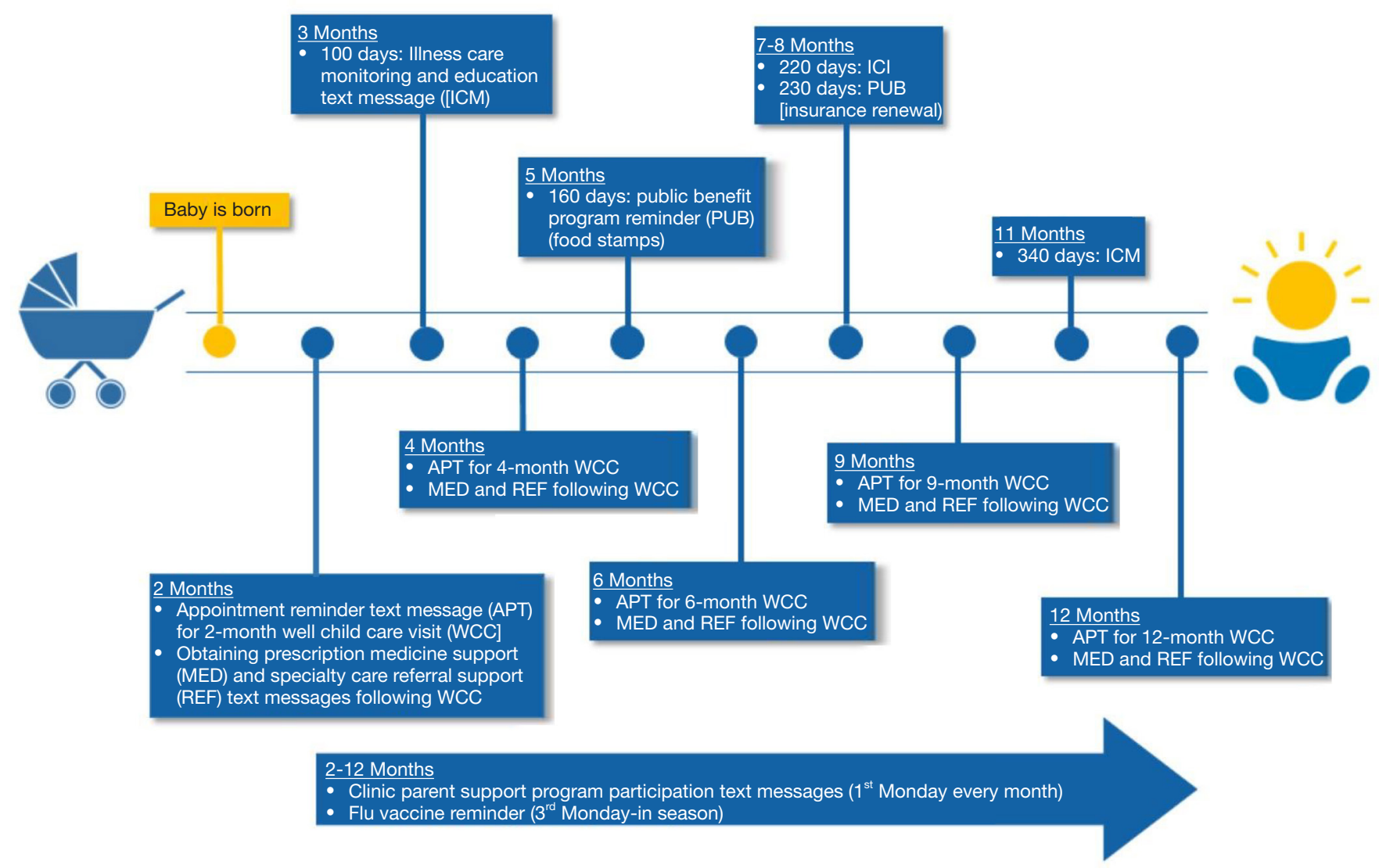

Figure 1 Timeline of intervention text message delivery.

in sociodemographics between those in the high and low engagement categories. All statistical analyses were conducted using STATA/SE Version 15.1 (StataCorp LP, College Station, TX).

\section{Results}

A total of 79 families were assigned to the text message intervention and comprised the study sample for analysis. Parents had a mean age of 30.1 years (SD 6.1 years) and had spent a mean of 7.5 years (SD 5.1 years) in the U.S. Nearly all $(\mathrm{n}=77,97.5 \%)$ parents reported limited English proficiency and the majority $(n=50,63.3 \%)$ had an education level below high school diploma or GED. Most parents ( $\mathrm{n}=67,84.8 \%$ ) were at risk for limited health literacy based on the NVS (Table 2).

\section{Text message volume and usability}

Participants received a mean of 45.0 (SD: 8.5) messages per participant (Table 3). The majority of messages received were part of interactive message sequences; the mean number of push messages per participant was 8.4 (SD: 2.4). Participants sent a mean of 15.5 messages per participant. 90 error messages were sent, which was $2.5 \%$ of all messages sent by all participants. Two-thirds (65.8\%) of participants sent at least one text message that generated a system error message. Among the subset of participants that sent any error message, $7.3 \%$ of messages they sent generated a system error message.

\section{Interactive text message sequence usability and engagement}

Table 4 describes participants' interactions with the text message sequences sent during the intervention. Four participants $(5 \%)$ did not respond to any sequences throughout the course of the intervention. Among interactive messages, participants' sequence completion rates were highest and median response time was lowest for APT sequences. Response rates to the first message in 
Table 2 Parent and child baseline characteristics

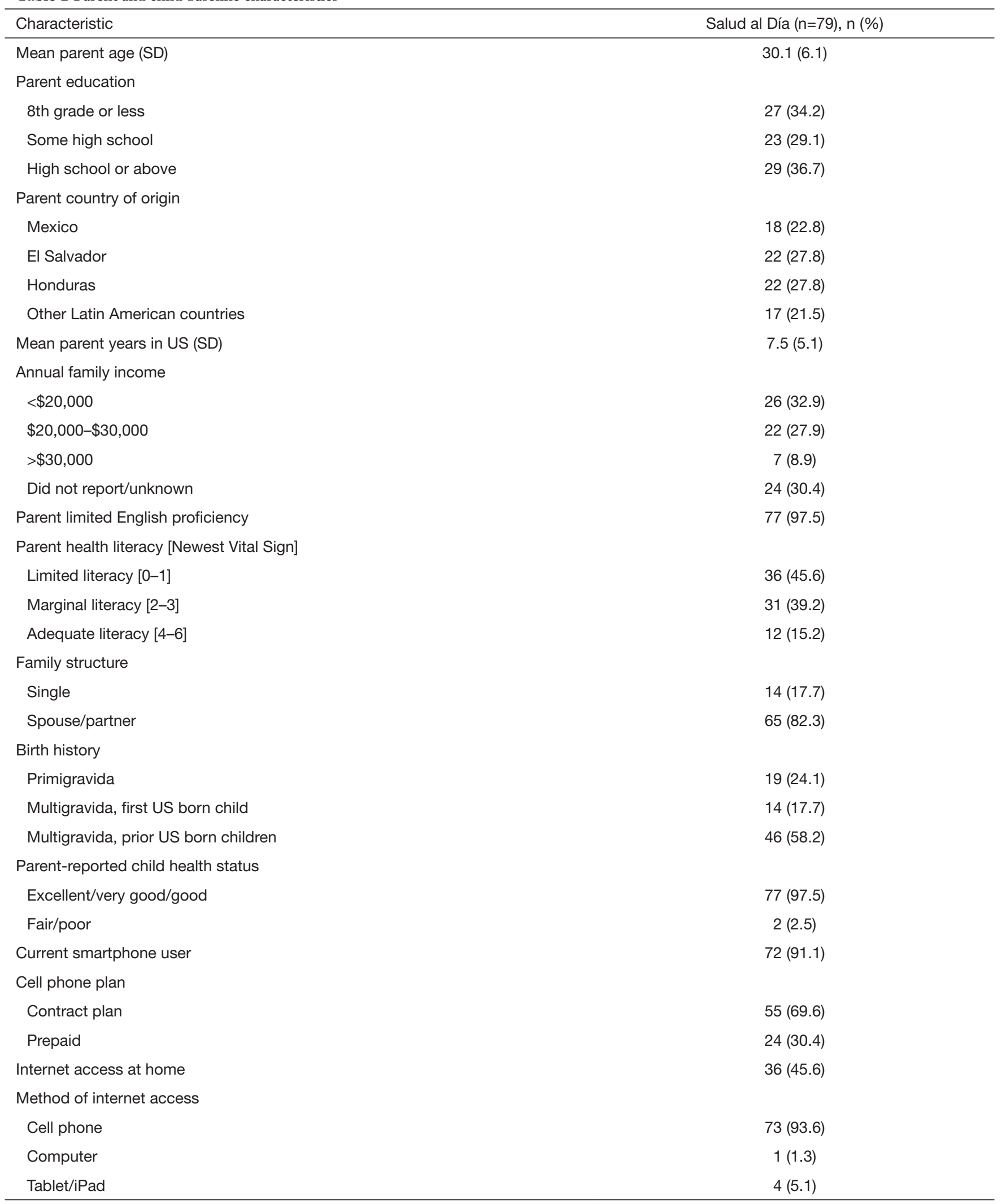


Table 3 Text message volume and usability by participants $(\mathrm{n}=79)$

\begin{tabular}{lcc}
\hline Measure & Total & Mean (SD) per participant \\
\hline Number of text messages received by participants & 3,560 & $45.0(8.5)$ \\
Number of push messages received by participants & 492 & $6.2(2.2)$ \\
Number of text messages sent by participants & 1,226 & $15.5(6.7)$ \\
$\%$ of participants that sent a text message that generated system error message & $65.8 \%$ & $\mathrm{~N} / \mathrm{A}$ \\
Number of text messages sent by participants that generated system error message & 90 & $1.7(1.4)$ \\
\hline
\end{tabular}

Table 4 Interactive text message sequence detail and participant $(n=79)$ response characteristics

\begin{tabular}{|c|c|c|c|c|}
\hline Text sequence content & $\begin{array}{l}\text { Mean (SD) total sequences } \\
\text { received by each participant }\end{array}$ & $\begin{array}{c}\text { Response rate to initial } \\
\text { message in sequence (\%) }\end{array}$ & $\begin{array}{l}\text { Median (IQR) response } \\
\text { time to initial message in } \\
\text { sequence }(\mathrm{min})\end{array}$ & $\begin{array}{c}\text { Sequence completion } \\
\text { rate }(\%)\end{array}$ \\
\hline $\begin{array}{l}\text { Obtaining prescription } \\
\text { medicine support (MED) }\end{array}$ & $4.7(0.8)$ & 71.8 & 9.7 (39.0) & 63.0 \\
\hline $\begin{array}{l}\text { Illness care } \\
\text { management (ICM) }\end{array}$ & $2.9(0.3)$ & 55.0 & $11.6(55.6)$ & 52.4 \\
\hline Push messages & $6.2(2.2)$ & N/A & $\mathrm{N} / \mathrm{A}$ & N/A \\
\hline
\end{tabular}

${ }^{\dagger}$, this is the initial message in the sequence for each of these text sequences. Number of messages in each sequence varied by participant response.

a sequence was highest for the MED sequences. Sequence completion was lowest for ICM sequences. Median response times were longest for REF sequences. Sequence completion over time was most variable for APT sequences (Figure 2). Among APT sequences, the highest completion rates were at 6 months. Sequence completion rates were less variable for MED and REF sequences.

When the sample was stratified by engagement quartiles, we found participants in the high engagement quartile completed a mean of $88.3 \%$ ( $\mathrm{SD}=0.05$ ) of sequences. Participants in the low engagement quartile completed a mean of $26.0 \%$ ( $\mathrm{SD}=0.18$ ) of sequences. Among participant sociodemographics, only parent education was significantly different between participants in the high and low engagement categories. $73.7 \%$ of participants in the high engagement had completed at least high school compared with $20.0 \%$ in the low engagement category $(\mathrm{P}=0.002)$. There were no significant differences in median response time to message sequences of any type by engagement category.

\section{Text message acceptability}

Of the 58 participants that responded to the midpoint survey, $93.1 \%$ rated the text messages as very easy/easy to use. Table 5 describes the acceptability of the text message intervention, assessed during a follow-up survey with 72 intervention participants. $97.2 \%$ of participants responded strongly agree/agree that the text messages helped them to remember their appointments at the clinic. Fewer participants $(65.3 \%)$ reported that the text message sequences helped them with obtaining medications or completing referrals or studies their child's pediatrician may have ordered. Nearly all participants $(95.8 \%)$ endorsed that the text messages helped them feel more connected to the clinic. $94.4 \%$ endorsed that the text messages made them feel that there was someone at the clinic who could help them if needed.

\section{Discussion}

An automated text messaging intervention (TMI) was 


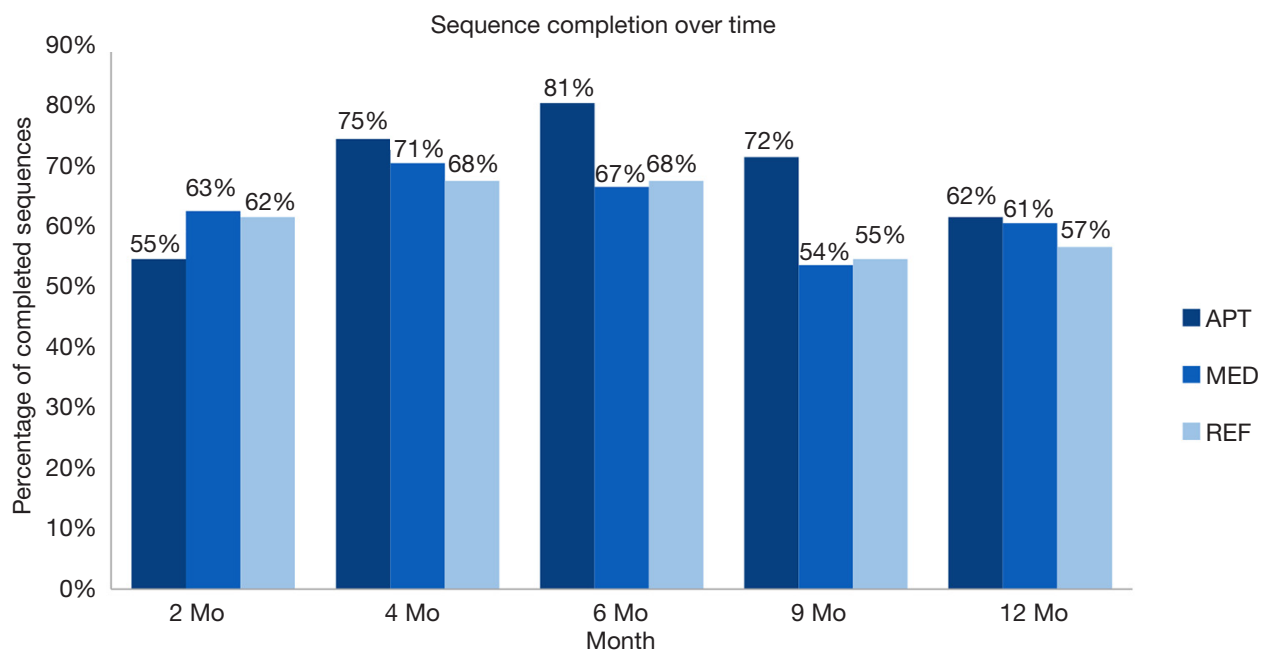

Figure 2 Sequence completion over time. APT, appointment reminder; MED, obtaining prescription medicine support; REF, specialty care referral support.

Table 5 Evaluation of text message acceptability

\begin{tabular}{|c|c|}
\hline Statement & $\begin{array}{l}\text { Acceptability statement endorsement } \\
\quad(\text { agree/strongly agree })^{\dagger}, \mathrm{n}(\%)\end{array}$ \\
\hline \multicolumn{2}{|l|}{ Care and appointment adherence } \\
\hline The text messages helped me ensure that my child received all vaccines & $61(84.7)$ \\
\hline The text messages made it easier to make appointments with the clinic & $64(88.9)$ \\
\hline $\begin{array}{l}\text { The text messages made it easier to make referral appointments with specialists/appointments for } \\
\text { special tests }\end{array}$ & $47(65.3)$ \\
\hline \multicolumn{2}{|l|}{ Communication and clinic experience } \\
\hline The text messages made me feel more connected to the clinic & $69(95.8)$ \\
\hline The text messaged helped me improve my communication with my child's doctor & $59(81.9)$ \\
\hline \multicolumn{2}{|l|}{ Application of information } \\
\hline The text messages helped me obtain information for my child's health insurance & $51(70.8)$ \\
\hline The text messages helped me obtain food stamps for my child & $22(30.6)$ \\
\hline
\end{tabular}

\footnotetext{
${ }^{\dagger}$ All responses based on a 5 -item Likert scale.
}

feasible and showed high usability and interactivity in a low-income LEP Latino population in a pediatric primary care setting. The TMI also had high levels of acceptability: the majority of participants reported that the text messages helped them make and remember appointments, receive vaccinations for their children, and feel more connected with the clinic. We found an association between lower engagement with the TMI and lower parental educational 
attainment when we stratified the sample by engagement category. Lower educational attainment and limited health literacy are established barriers to the effectiveness of health and healthcare interventions. The majority of parents in this trial had below a high school education and were at risk of limited health literacy yet the intervention was still feasible, usable and acceptable. Our findings support the use of TMIs to address health and healthcare disparities among immigrant Latino families with LEP.

Our findings and other studies demonstrate that TMIs are an effective way to reach low-income populations (36). Usability and acceptability of the TMIs in our study was comparable to that in populations with higher education or health literacy and other TMIs implemented in demographically similar populations $(23,24)$. This suggests that TMIs are able to address some of the challenges to health promotion intervention effectiveness related to limited education and health literacy. The high usability of the TMI may have been due in part to its user-centered design, which has been shown to lead to more favorable usability and acceptability profiles compared with digital health interventions (DHIs) that are developed in one context and then scaled to others $(37,38)$. Co-development with LEP Latino families helped ensure that text messages were relevant, met cultural expectations for communication and could be easily understood and responded to. Involving end-users and community stakeholders in the cultural and linguistic tailoring of interventions to optimize utilization and engagement may be critical to closing the gap in digital engagement between healthcare providers and low-income and racial/ethnic minority populations $(39,40)$. While this intervention demonstrated that contextual tailoring can lead to effective interventions, the degree and specificity of tailoring that is needed remains unknown. Understanding how much tailoring is necessary for intervention effectiveness is needed to promote feasibility of use of these programs at scale in large systems serving diverse populations. As platforms evolve there may be technologic capacity for more variability across participants within a single digital health tool. This would allow for some context specific tailoring as well as promote feasibility for health systems.

Our findings demonstrate some key accommodations that should be considered in TMIs and other digital health tools to promote engagement of LEP Latino populations. Response times to sequence messages varied widely in our study, which may have been due to variable access to the cellular phone receiving messages. The need to accommodate this variability suggests future interventions should consider building algorithms for this population that allow for longer response times. We did not employ response prompting (e.g., automatically sending a followup message if participants failed to respond to the initial message), a strategy which may have improved response rates. Results from our study showed that, upon responding to the initial sequence message, participants were quick to respond to subsequent messages, however. This finding highlights the importance of minimizing the delivery time of automated response messages to maximize participant engagement with the text message sequences. In our system, median delivery time of automated messages was less than 30 seconds, which facilitated high levels of engagement with message sequences. As technology improves, server cycling times ought to be enhanced to minimize delays in automated message responses and maximize end-user engagement with these systems.

Certain study limitations deserve mention. First, our study was conducted at a single clinic, and our study population consisted of a small sample that may not be representative of other outpatient pediatric settings. Parents in our sample varied in their age and country of origin, which may support generalizability to a wider Latino population, however. Second, the emocha ${ }^{\circledR}$ mHealth platform utilized the application programming interface (API) of a third-party service to send and receive text messages. Our study team did not have access to this thirdparty console, thus we were unable to monitor real-time messaging errors (e.g., message send failure as a result of a changed phone number or disconnected phone line). Access to these real-time updates might have created opportunities to re-engage participants who became unable to receive the intervention text messages due to lapses in cellular phone service. Despite this limitation, we observed high levels of engagement with the TMI over time, which suggests that this did not pose a significant barrier in our study. Third, emocha $^{\circledR}$, the platform used in our study, was different from the vendor used by the health system to deliver appointment reminders by text message. We would not have had the design flexibility afforded by the third-party vendor with the health system vendor. The cost of using a different vendor was covered by the study grant, but this was not an expense the study clinic could have otherwise covered. For sustainability, use of the health system vendor would be preferred. As programming flexibility with commercial vendors who serve large health systems increases, use of these vendors for future interventions may be possible. 
There is often cost sharing, however, for these modifications between health systems and individual clinics. The ability to sustain this intervention and disseminate to other clinics is limited by the use of a third-party vendor and potential cost sharing with use of the health system vendor. In general, programming and development costs are a critical barrier to widespread use of TMIs that are tailored for vulnerable populations in routine clinical care.

In conclusion, our study showed that an automated TMI was feasible, usable and acceptable among a low-income immigrant LEP Latino population in a pediatric primary care setting. These findings highlight the potential for mHealth tools to better engage LEP families in accessing and using primary care, which may ultimately help to reduce the health disparities experienced by this population. Next steps for this work include implementation and dissemination, with the focus shifting from research to routine clinical care. In the implementation phase, it will be critical that vendors allow sufficient flexibility in the design and usability of their systems to tailor to vulnerable populations, and that there is the ability to share programming and development costs across healthcare systems that serve similar populations. Failures in implementation and dissemination may widen the digital divide and perpetuate health disparities for these populations.

\section{Acknowledgments}

We extend sincere thanks and appreciation for the members of the Latino Family Advisory Board, without whom this project would not have been possible.

Funding: This study was supported by the Gordon and Betty Moore Foundation.

\section{Footnote}

Conflicts of Interest: Both authors have completed the ICMJE uniform disclosure form (available at http://dx.doi. org/10.21037/mhealth.2020.01.06). The authors have no conflicts of interest to declare.

Ethical Statement: The authors are accountable for all aspects of the work in ensuring that questions related to the accuracy or integrity of any part of the work are appropriately investigated and resolved. The study was conducted in accordance with the Declaration of Helsinki (as revised in 2013). The Institutional Review Board at Johns Hopkins Medicine approved this study. Informed consent was obtained from each participant.

Open Access Statement: This is an Open Access article distributed in accordance with the Creative Commons Attribution-NonCommercial-NoDerivs 4.0 International License (CC BY-NC-ND 4.0), which permits the noncommercial replication and distribution of the article with the strict proviso that no changes or edits are made and the original work is properly cited (including links to both the formal publication through the relevant DOI and the license). See: https://creativecommons.org/licenses/by-nc-nd/4.0/.

\section{References}

1. Flores $\mathrm{G}$ and the Committee on Pediatric Research. Technical report--racial and ethnic disparities in the health and health care of children. Pediatrics 2010;125:e979-1020.

2. Flores G, Olson L, Tomany-Korman SC. Racial and ethnic disparities in early childhood health and health care. Pediatrics 2005;115:e183-93.

3. Flores G, Tomany-Korman SC. Racial and ethnic disparities in medical and dental health, access to care, and use of services in US children. Pediatrics 2008;121:e286-98.

4. Flores G, Tomany-Korman SC. The language spoken at home and disparities in medical and dental health, access to care, and use of services in US children. Pediatrics 2008;121:e1703-14.

5. DeCamp LR, Choi H, Davis MM. Medical home disparities for Latino children by parental language of interview. J Health Care Poor Underserved 2011;22:1151-66.

6. Brousseau DC, Hoffmann RG, Yauck J, et al.Disparities for Latino children in the timely receipt of medical care. Ambul Pediatr 2005;5:319-25.

7. Kubicek K, Liu D, Beaudin C, et al. A profile of nonurgent emergency department use in an urban pediatric hospital. Pediatr Emerg Care 2012;28:977-84.

8. Yin HS, Sanders LM, Rothman RL, et al. Assessment of health literacy and numeracy among Spanish-Speaking parents of young children: validation of the Spanish Parental Health Literacy Activities Test (PHLAT Spanish). Acad Pediatr 2012;12:68-74.

9. DeCamp LR, Bundy DG. Generational status, health insurance, and public benefit participation among low-income Latino children. Matern Child Health J 2012;16:735-43. 
10. Ngui EM, Flores G. Satisfaction with care and ease of using health care services among parents of children with special health care needs: the roles of race/ethnicity, insurance, language, and adequacy of family-centered care. Pediatrics 2006;117:1184-96.

11. DeCamp LR, Kieffer E, Zickafoose JS, et al. The voices of limited English proficiency Latina mothers on pediatric primary care: lessons for the medical home. Matern Child Health J 2013;17:95-109.

12. NORC at the University of Chicago. Briefing Paper: Understanding the Impact of Health IT in Underserved Communities and those with Health Disparities. University of Chicago 2010:1-69.

13. López L, Green AR, Tan-McGrory A, et al. Bridging the digital divide in health care: the role of health information technology in addressing racial and ethnic disparities. Jt Comm J Qual Patient Saf 2011;37:437-45.

14. Kruse CS, Bolton K, Freriks G. The effect of patient portals on quality outcomes and its implications to meaningful use: a systematic review. J Med Internet Res 2015;17:e44.

15. Irizarry T, DeVito Dabbs A, Curran CR. Patient Portals and Patient Engagement: A State of the Science Review. J Med Internet Res 2015;17:e148.

16. Ketterer T, West DW, Sanders VP, et al. Correlates of patient portal enrollment and activation in primary care pediatrics. Acad Pediatr 2013;13:264-71.

17. Goel MS, Brown TL, Williams A, et al.Disparities in enrollment and use of an electronic patient portal. J Gen Intern Med 2011;26:1112-6.

18. Poorman E, Gazmararian J, Parker RM, et al. Use of text messaging for maternal and infant health: a systematic review of the literature. Matern Child Health J 2015;19:969-89.

19. Lin CL, Mistry N, Boneh J, et al. Text message reminders increase appointment adherence in a pediatric clinic: A randomized controlled trial. Int J Pediatr 2016;2016:8487378.

20. Stockwell MS, Kharbanda EO, Martinez RA, et al.Effect of a text messaging intervention on influenza vaccination in an urban, low-income pediatric and adolescent population: a randomized controlled trial. JAMA 2012;307:1702-8.

21. O'Leary ST, Lee M, Lockhart S, et al. Effectiveness and cost of bidirectional text messaging for adolescent vaccines and well care. Pediatrics 2015;136:e1220-7.

22. Ladley A, Hieger AW, Arthur J, et al. Educational text messages decreased Emergency Department utilization among infant caregivers: A randomized trial. Acad Pediatr
2018;18:636-41.

23. Text4baby Data and Evaluation. Text4baby: Reaching Latinas with important health information. Available online: https://partners.text4baby.org/templates/beez_20/ images/2014/t4bserviceinspanishfactsheet12814.pdf. Accessed 10 January 2020.

24. Aguilera A, Muñoz RF. Text messaging as an adjunct to CBT in low-income populations: A usability and feasibility pilot study. Prof Psychol Res Pr 2011;42:472-8.

25. Head KJ, Noar SM, Iannarino NT, et al. Efficacy of text messaging-based interventions for health promotion: a meta-analysis. Soc Sci Med 2013;97:41-8.

26. Ramirez M, Wu S, Beale E. Designing a Text Messaging Intervention to Improve Physical Activity Behavior Among Low-Income Latino Patients With Diabetes: A DiscreteChoice Experiment, Los Angeles, 2014-2015. Prev Chronic Dis 2016;13:E171.

27. Dick JJ, Nundy S, Solomon MC, et al. Feasibility and usability of a text message-based program for diabetes selfmanagement in an urban African-American population. J Diabetes Sci Technol 2011;5:1246-54.

28. emocha mobile health. Available online: www.emocha. com. Accessed: 10 January 2020.

29. Rivet Amico K. A situated-Information Motivation Behavioral Skills Model of Care Initiation and Maintenance (sIMB-CIM): an IMB model based approach to understanding and intervening in engagement in care for chronic medical conditions. J Health Psychol 2011;16:1071-81.

30. Bright Futures/American Academy of Pediatrics, Recommendations for Preventive Pediatric Health Care, 2017. Available online: https://www.aap.org/enus/Documents/periodicity_schedule.pdf. Accessed: 15 September 2019

31. Vickers M, Anderson B, Dworetzky B, et al. Traducción al español de Monica Bernot. Bright Futures - Guía para las Familias - Criando bebés, niños y adolescentes saludables. Albuquerque, NM: Family Voices, 2014.

32. REDCap Research Electronic Data Capture. Available online: http://project-redcap.org. Accessed: 10 January 2020.

33. Harris PA, Taylor R, Thielke R, et al. Research electronic data capture (REDCap)--a metadata-driven methodology and workflow process for providing translational research informatics support. J Biomed Inform 2009;42:377-81.

34. US Census Bureau. Language Use. Available online: https://www.census.gov/topics/population/language-use/ about.html. Accessed: 10 January 2020 
35. Pfizer Newest Vital Sign. Available online: https://www. pfizer.com/health/literacy/public-policy-researchers/nvstoolkit. Accessed: 15 September 2019.

36. Anderson-Lewis C, Darville G, Mercado RE, et al. mHealth Technology Use and Implications in Historically Underserved and Minority Populations in the United States: Systematic Literature Review. JMIR Mhealth Uhealth 2018;6:e128.

37. Tarver WL, Haggstrom DA. The Use of Cancer-Specific Patient-Centered Technologies Among Underserved Populations in the United States: Systematic Review. J Med Internet Res 2019;21:e10256.

38. Mayberry LS, Berg CA, Harper KJ, et al. The Design,

\section{doi: $10.21037 /$ mhealth.2020.01.06}

Cite this article as: Silverman-Lloyd LG, Dominguez Cortez J, Godage SK, Valenzuela Araujo D, Rivera T, Polk $\mathrm{S}$, DeCamp LR. Immigrant Latino parents demonstrated high interactivity with pediatric primary care text messaging intervention. mHealth 2020;6:45.
Usability, and Feasibility of a Family-Focused Diabetes Self-Care Support mHealth Intervention for Diverse, Low-Income Adults with Type 2 Diabetes. J Diabetes Res 2016;2016:7586385.

39. Bock BC, Rosen RK, Barnett NP, et al. Translating Behavioral Interventions Onto mHealth Platforms: Developing Text Message Interventions for Smoking and Alcohol. JMIR mHealth uHealth 2015;3:e22.

40. Huberty J, Rowedder L, Hekler E, et al. Development and design of an intervention to improve physical activity in pregnant women using Text4baby. Transl Behav Med 2016;6:285-94. 